\title{
Design of a 6-DOF upper limb rehabilitation exoskeleton with parallel actuated joints
}

\author{
Yanyan Chen, Ge Li, Yanhe Zhu", Jie Zhao and Hegao Cai \\ State Key Laboratory of Robotics Institute, Harbin Institute of Technology University, Harbin, \\ Heilongjiang Province 150006, China
}

\begin{abstract}
In this paper, a 6-DOF wearable upper limb exoskeleton with parallel actuated joints which perfectly mimics human motions is proposed. The upper limb exoskeleton assists the movement of physically weak people. Compared with the existing upper limb exoskeletons which are mostly designed using a serial structure with large movement space but small stiffness and poor wearable ability, a prototype for motion assistance based on human anatomy structure has been developed in our design. Moreover, the design adopts balls instead of bearings to save space, which simplifies the structure and reduces the cost of the mechanism. The proposed design also employs deceleration processes to ensure that the transmission ratio of each joint is coincident.
\end{abstract}

Keywords: Upper limb, parallel actuated joints, exoskeleton, rehabilitation

\section{Introduction}

In the current society, the aging process is accelerating. Stroke is one of the diseases that commonly happen among elder people. The desease is characterized with rapid onset, fast development, and high rate of disability. It is especially common in an aging society with pressure and challenges. Due to the fact that its various sequelae cause a high rate of disability, the rehabilitation work is particularly important. The upper limb rehabilitation exoskeleton is used to help patients with the rehabilitation training. For the traditional rehabilitation therapists, the training process is monotonous with frequent repetition and requires a considerable amount of physical consumption. Meanwhile it is hard to ensure the stability and continuity of rehabilitation training. As an alternative, the exoskeleton rehabilitation is a more suitable and low cost method. In this sense, it is essential to develop a reliable rehabilitation exoskeleton. Different from the virtual reality rehabilitation system [1], the exoskeleton can be used not only in training but also in daily life. Compared with lower extremity [2], the movement of human upper limb is flexible, complex, and wide-ranged. Therefore, the complete duplication of human upper limb structure is very difficult. However, we have been successfully developing upper limb

\footnotetext{
${ }^{*}$ Corresponding author: Yanhe Zhu, State Key Laboratory of Robotics Institute, Harbin Institute of Technology University, HIT University Science Park Robotics Institute 203, Building C1, Room 203, Science Square, Yikuang Street No. 2, Nangang District, Harbin, Heilongjiang Province 150006, China. Tel.: 13074594165; Fax: +86-045186414538; E-mail: yhzhu@hit.edu.cn.
} 
exoskeletons to assist physically weak people including elderly people, handicapped people, or even children with hemiplegia [3].

Many universities and research institutes have developed different kinds of upper limb rehabilitation exoskeletons [4-14]. Gupta et al. [4] and Klein et al. [5] proposed exoskeletons with parallel link mechanism. Sasaki et al. developed a one degree of freedom (DOF) assistant exoskeleton with soft actuated for rehabilitation training. The exockeleton had a simple construction which did not support full exercises for upper limb joints. Jiafan et al. [6] developed ZJUESA, a 6-DOF exoskeleton, and it was also actuated by a pneumatic system. Saga University developed an exoskeleton which could change the center of rotation of the shoulder joint, making it more similar to human, but the workspace was limited. Manus, designed by MIT, was a link mechanism. By holding the end-effector, patients could achieve two-dimensional rehabilitation training for shoulder and elbow joints under the robot traction. Perry and Rosen [9] designed a 7-DOF serial exoskeleton which combined joints and negligible backlash in seven force-reflecting articulations. However, there was a wrist joint bias problem need to be solved for the exoskeleton. Additionally, the shoulder joint was away from the human body. Kobayashi et al. [13] proposed a wearable robot but this mechanism had limited strength. Hocoma [14] developed ArmeoSpring, an upper limb exoskeleton that can cooperate with virtual reality games in rehabilitation on training. Zhang jiafan [15] designed a 9-DOF flexible pneumatic upper limb exoskeleton including the 3RPS parallel mechanism and the slip rings. Due to the usage of cylinder, it can imitate the motion of human muscles, but it also cannot reach a wide workspace.

Most present exoskeletons are serial structures. The serial mechanism allows the exoskeleton to have simple structures, large movement spaces, and simple control system. But compared with the parallel mechanism, serial structure has small stiffness, low accuracy and weak carrying capacity. In this study, a prototype 6-DOF exoskeleton with parallel actuated joints for motion assistance based on human anatomy structure is developed. In the design, we also adopted deceleration processes and a trench structure to place balls rather than using bearings to perfect the system.

\section{Human upper limb anatomy}

\subsection{Human upper limb structure}

The shoulder involves the most complex, flexible, and determinant mechanism of the human upper limb, comprising the thorax, clavicle, scapula and humerus, and four articulations: the glenohumeral (GH), acromioclavicular (AC), sternoclavicular (SC), and scapulothoracic (ST) joints [16-18]. Upper limb humerus and scapula are connected through the shoulder joint. It can be regarded as a ball-andsocket joint of 3-DOF that comprises the ball-shape cavitas glenoidalis. The shoulder joint has a big humeral head and a small glenoid cavity. Joint capsule is thin and slack and the inferior wall of capsule is thin.

Elbow is an important joint that connects forearm and upper arm. The elbow structure is formed between the forearm by ulna and radius and the humerus. Humeroradial joint is a concave spherical joint and the ulnohumeral joint is a hinge joint.

Wrist connects the forearm and the hand. As a composite joint, it includes the arc joint surface of the radial bottom, articular disc of ulnar bottom, and metacarpale, also known as the radiocarpal joint (See Figure 1). 


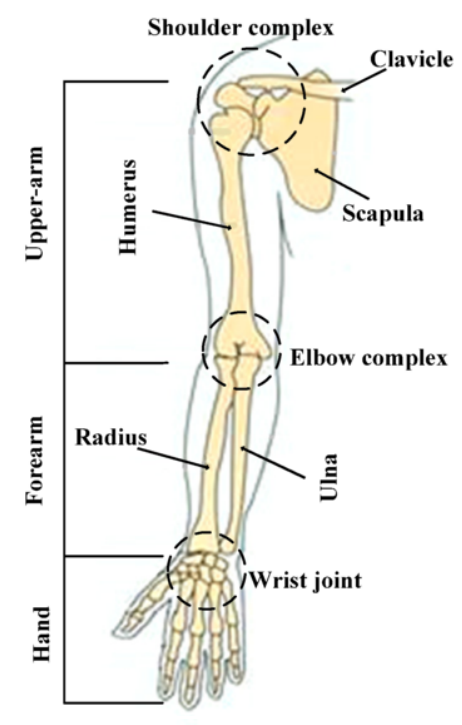

Fig. 1. The human upper limb anatomy structure.

\subsection{Human upper limb motion}

Assuming negligible joint translations, the shoulder joint is typically modeled as ideal 3-DOF balland-socket joints. Moreover, position of the center of rotation of the shoulder joint changes with the movement of upper limb. The articulation between thorax and scapula is formed by an area on the posterior wall of the thorax, called the scapulothoracic gliding plane, over which the anterior wall of the scapula is restricted to move. In most models, the ST gliding plane is incorporated by assuming a constant distance between the medial border of the scapula and the posterior side of the thorax, which is usually approximated by an ellipsoid [19,20]. In the real human motion, the 2-DOF of SC can drive the shoulder and upper limb up and down around the shoulder joint shrug and stretch. In addition, when the SC joint drives $\mathrm{AC}$ joint and $\mathrm{GH}$ joint motion, $\mathrm{AC}$ joint plays a coordinating role. While the $\mathrm{AC}$ joint acts as a passive joint, it cannot move alone. However it acts like a cord in the movement of other relative joints. At the same time, due to the close physical location of the AC joint and $\mathrm{GH}$ joint as well as the fact that both of them have 3 DOFs, we can simplified the two joints as one with a 3DOF shoulder joint [21].

When the ulnohumeral joint rotates, ulna drives radius to rotate around the rotation axis of humeroulnar joint, which is the elbow flexion movement. When the humeroradial joint rotates around the longithdinal axis, radius rotates around the ulna and formed a pronation motion. Because of the constraint of longitudinal ridge, coronary fossa, radial fossa, and radial ligament, elbow movement stays in a suitable range.

The wrist joint possesses two DOF: flexion/extension and radial/ulnar deviation. Wrist motions are generated around an instantaneous center. The path of the centrode is small. However, conventionally, the displacement of the instantaneous center of rotation is ignored and the rotation axes for the flexion/extension and ulna/radial deviation are considered to be fixed [22]. The movement ranges about the upper limb joints are shown in Table 1. 
Table 1

The movement range of each DOF [22-24]

\begin{tabular}{ll}
\hline Degrees of freedom & Movement range \\
\hline Shoulder flexion/extension & $150^{\circ}-180^{\circ} / 40^{\circ}-50^{\circ}$ \\
Shoulder abduction/adduction & $180^{\circ} / 30^{\circ}-40^{\circ}$ \\
Shoulder internal/external rotation & $70^{\circ}-95^{\circ} / 40^{\circ}-70^{\circ}$ \\
Elbow flexion/extension & $135^{\circ}-140^{\circ} / 0^{\circ}$ \\
Forearm supination/pronation & $85^{\circ}-90^{\circ} / 70^{\circ}-90^{\circ}$ \\
Wrist flexion/extension & $73^{\circ} / 70^{\circ}$ \\
Wrist radial deviation/ulnar deviation & $27^{\circ} / 27^{\circ}$ \\
\hline
\end{tabular}

\section{Exoskeleton design}

\subsection{The distribution of DOF}

In order to mimic human motions, we designed a differential mechanism of bevel gear transmission which can generate two rotational DOF. There are two gear rings and two support rings in the mechanism. Both of the gear rings have two parts: a straight gear and a bevel gear ring. The straight gear meshing with the straight gear ring can drive the rotation of gear ring. The gear rings then drive the bevel gears, which is connected with the upper limb. The model of upper limb and the exoskeleton are shown in Figure 2. In the model, $\mathrm{x}$-axis is the axis of shoulder internal/external rotation, $y$-axis is the axis of shoulder abduction/adduction rotation, and z-axis is the axis of shoulder flexion/extension.

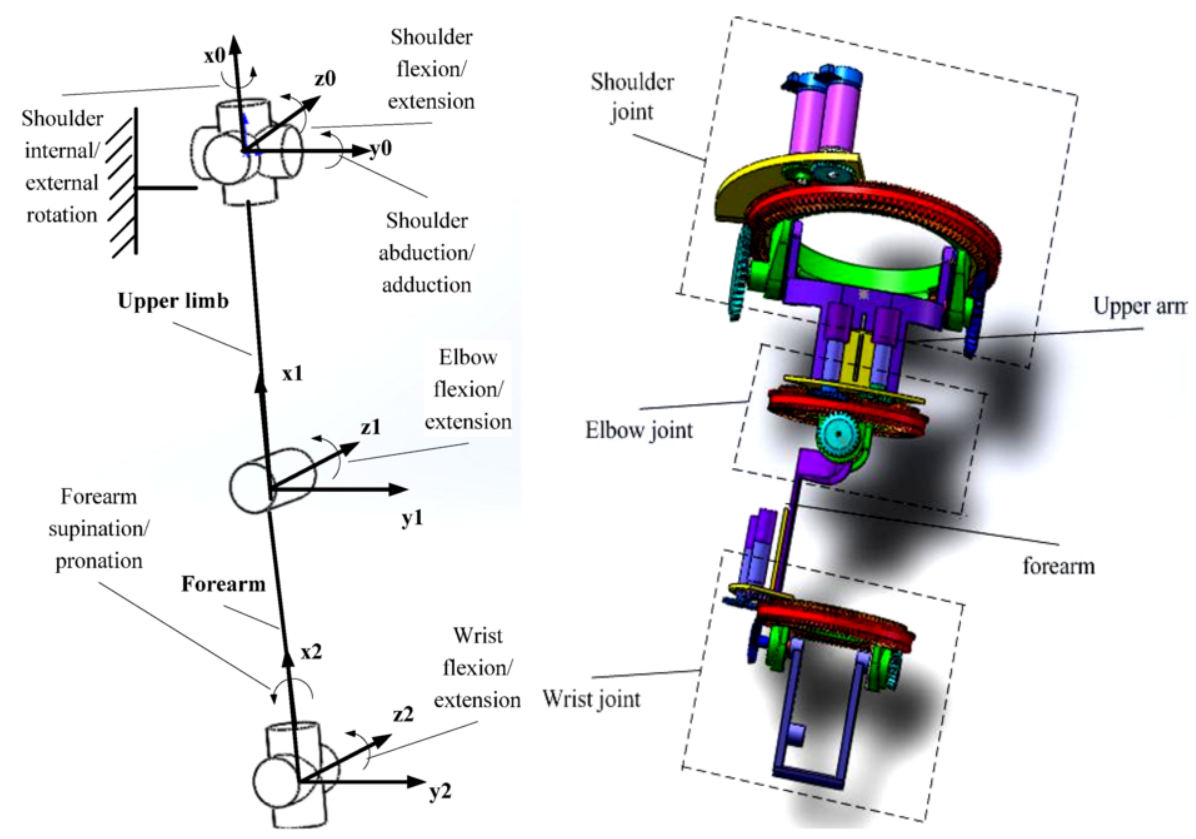

Fig. 2. Model of upper limb exoskeleton and the exoskeleton. 
The structures of the elbow and wrist joint also use this mechanism to achieve elbow flexion/extension, wrist flexion/extension, and forearm pronation/supination.

\subsection{Upper limb exoskeleton movement analysis}

When the two gear rings rotate in the same direction at the same speed, the following conditions hold:

$$
\theta_{1}=\theta_{2}, \beta_{1}=-\beta_{2}, \alpha_{1}=\alpha_{2}=0
$$

These conditions generate the motion of shoulder flexion/extension. When the two gear rings rotate in reverse at the same speed, the following conditions hold:

$$
\theta_{1}=-\theta_{2}, \beta_{1}=\beta_{2}, \alpha_{1}=\alpha_{2}=\theta_{1}
$$

These conditions generate the motion of shoulder abduction/adduction. When the upper arm is lifted horizontalto the shoulder, the axis of the gear ring coincides with the axis of upper arm and the flexion/extension DOF is changed into pronation/supination DOF. Variants of this configuration alignwith the $\mathrm{i}$-axis slightly upwards and/or towards the rear to optimize the range of motion for particular applications. Because rings of the elbow are placed on the upper arm, when the upper arm is singular in this configuration, the twisting of the rotary joint of elbow provides redundancy for shoulder. In terms of the wrist joint, considering that the axis of wrist radial deviation/ulnar deviation is normal to the axis of wrist flexion/extension, we turn the exoskeleton by $90^{\circ}$ and then can get the

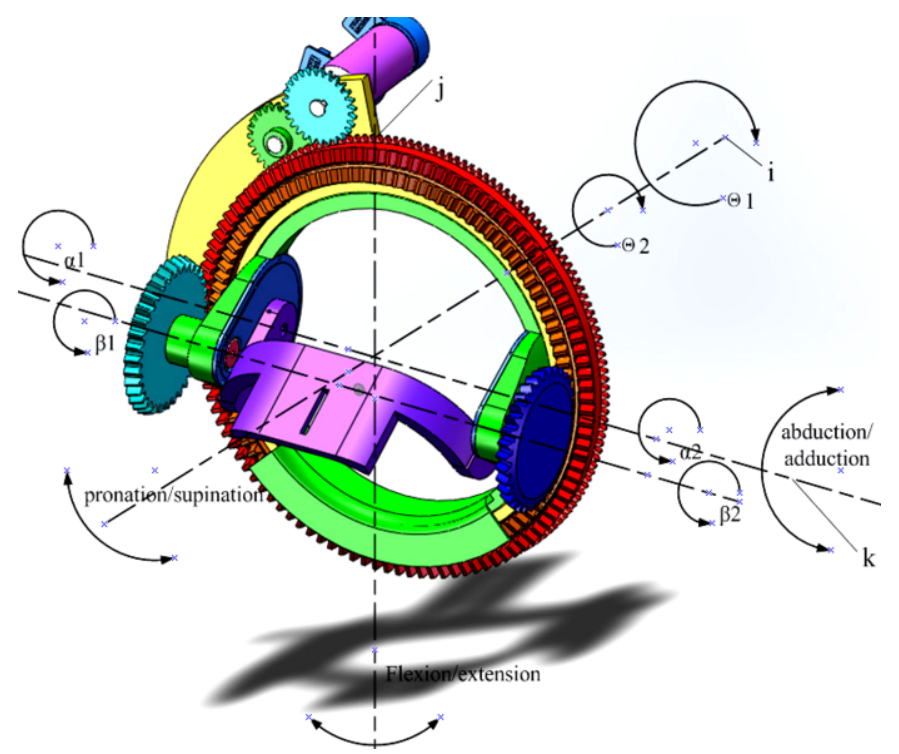

Fig. 3. Sketch of shoulder joint movement analysis. 

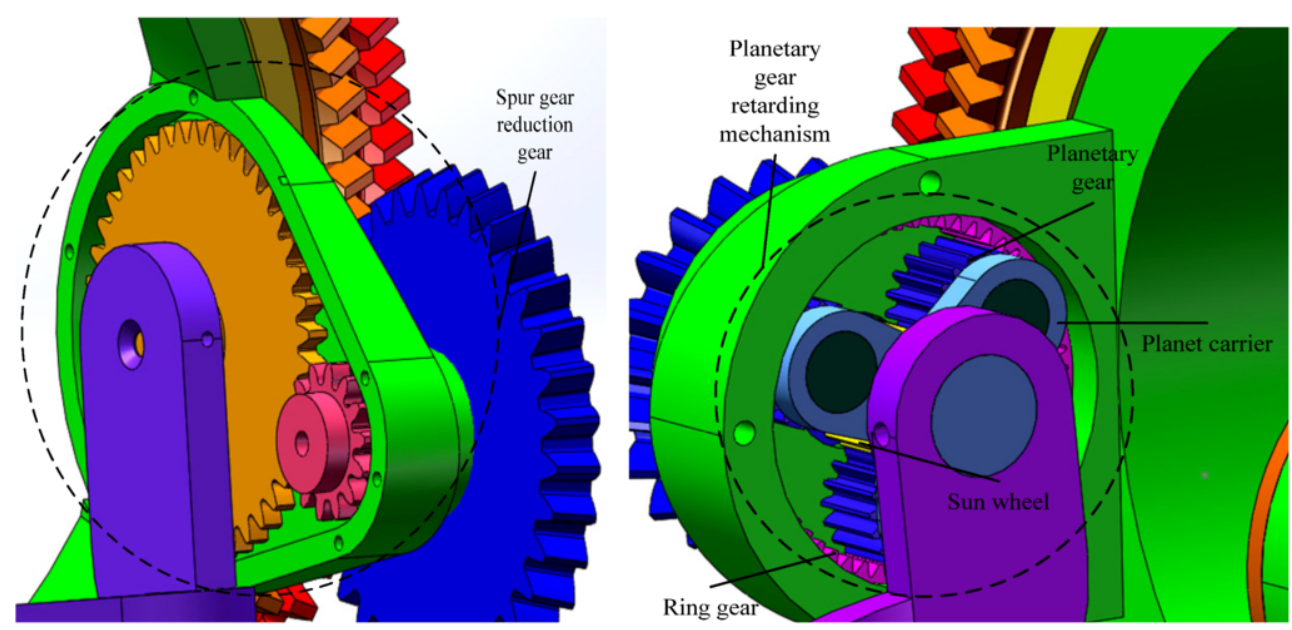

Fig. 4. Structure of deceleration processes.

DOF of wrist radial deviation/ulnar deviation from wrist flexion/extension. With the parallel structure we designed, the upper limb exoskeleton can also achieve relatively large workspace. Considering the exoskeleton only, each joint can reach a hemispherical space range, which is larger than the human upper limb motion. In the future work, a set of limit switches will be used to make sure the exoskeleton work in a reasonable safety space. Figure 3 shows the sketch of shoulder joint movements.

Between every two rings, we employed a trench structure to place balls rather than bearings. The balls save spaces, simplify the structure, and reduce the cost of the exoskeleton. In this design, we use different deceleration processes in shoulder and elbow to ensure that the transmission ratio of each DOF from the input to output is 1 . The structure of deceleration processes are shown in Figure 4.

\subsection{Kinematics analysis}

Defining the exoskeleton handle as the end point and the end point position as follows:

$$
T={ }^{0} A_{1} \bullet{ }^{1} A_{2} \bullet{ }^{2} A_{3}
$$

where $T$ is a transformation matrix and

$$
{ }^{0} A_{1}=T_{\mathrm{x}_{0},-l_{1}}=\left[\begin{array}{cccc}
1 & 0 & 0 & -l_{1} \\
0 & 1 & 0 & 0 \\
0 & 0 & 1 & 0 \\
0 & 0 & 0 & 1
\end{array}\right]
$$




$$
\begin{aligned}
& { }^{1} A_{2}=R_{z_{1}, \theta_{z_{1}}} \bullet T_{x_{1}, l_{2}}=\left[\begin{array}{cccc}
c \theta_{z_{1}} & -s \theta_{z_{1}} & 0 & 0 \\
s \theta_{z_{1}} & c \theta_{z_{1}} & 0 & 0 \\
0 & 0 & 1 & 0 \\
0 & 0 & 0 & 1
\end{array}\right] \bullet\left[\begin{array}{cccc}
1 & 0 & 0 & -l_{2} \\
0 & 1 & 0 & 0 \\
0 & 0 & 1 & 0 \\
0 & 0 & 0 & 1
\end{array}\right] \\
& { }^{2} A_{3}=R_{x_{2}, \theta_{x_{2}}} \bullet R_{z_{2}, \theta_{z_{2}}} \bullet T_{x_{2}, l_{3}}=\left[\begin{array}{cccc}
1 & 0 & 0 & 0 \\
0 & c \theta_{x_{2}} & -s \theta_{x_{2}} & 0 \\
0 & s \theta_{x_{2}} & c \theta_{x_{2}} & 0 \\
0 & 0 & 0 & 1
\end{array}\right] \bullet\left[\begin{array}{cccc}
c \theta_{z_{2}} & -s \theta_{z_{2}} & 0 & 0 \\
s \theta_{z_{2}} & c \theta_{z_{2}} & 0 & 0 \\
0 & 0 & 1 & 0 \\
0 & 0 & 0 & 1
\end{array}\right] \bullet\left[\begin{array}{cccc}
1 & 0 & 0 & -l_{3} \\
0 & 1 & 0 & 0 \\
0 & 0 & 1 & 0 \\
0 & 0 & 0 & 1
\end{array}\right]
\end{aligned}
$$

where $\theta_{z_{1}}$ is the rotation angle around $z_{1}$ - axis (other angles are the corresponding meaning); $l_{1}$ is the length of the upper limb; $l_{2}$ is the length of the forearm; $l_{3}$ is the distance between wrist joint and the end point.

Substitute Eqs. (2)-(4) into Eq. (1), we get:

$$
T=\left[\begin{array}{cccc}
c \theta_{z_{1}} c \theta_{z_{2}}-s \theta_{z_{1}} c \theta_{z_{2}} s \theta_{z_{2}} & -c \theta_{z_{1}} s \theta_{z_{2}}-s \theta_{z_{1}} c \theta_{x_{2}} c \theta_{z_{2}} & s \theta_{z_{1}} s \theta_{x_{2}} & -l_{3} c \theta_{z_{1}} c \theta_{z_{2}}+l_{3} s \theta_{z_{1}} c \theta_{x_{2}} s \theta_{z_{2}} \\
s \theta_{z_{1}} c \theta_{z_{2}}+c \theta_{z_{1}} c \theta_{x_{2}} s \theta_{z_{2}} & -s \theta_{z_{1}} s \theta_{z_{2}}+c \theta_{z_{1}} c \theta_{x_{2}} c \theta_{z_{2}} & -c \theta_{z_{1}} s \theta_{x_{2}} & -l_{3} s \theta_{z_{1}} c \theta_{z_{2}}-l_{3} c \theta_{z_{1}} c \theta_{x_{2}} s \theta_{z_{2}}-l_{2} s \theta_{z_{1}} \\
s \theta_{x_{2}} s \theta_{z_{2}} & s \theta_{x_{2}} c \theta_{z_{2}} & c \theta_{x_{2}} & -l_{3} s \theta_{x_{2}} s \theta_{z_{2}} \\
0 & 0 & 0 & 1
\end{array}\right]
$$

$x_{0}-y_{0}-z_{0}$ is a moving coordinate system which changes with the motion of upper limb.

$$
\begin{aligned}
R & =R_{x_{0}, \theta_{x_{0}}} \bullet R_{y_{0}, \theta_{y_{0}}} \bullet R_{z_{0}, \theta_{z_{0}}} \\
& =\left[\begin{array}{cccc}
1 & 0 & 0 & 0 \\
0 & c \theta_{x_{0}} & -s \theta_{x_{0}} & 0 \\
0 & s \theta_{x_{0}} & c \theta_{x_{0}} & 0 \\
0 & 0 & 0 & 1
\end{array}\right] \bullet\left[\begin{array}{cccc}
c \theta_{y_{0}} & 0 & s \theta_{y_{0}} & 0 \\
0 & 1 & 0 & 0 \\
-s \theta_{y_{0}} & 0 & c \theta_{y_{0}} & 0 \\
0 & 0 & 0 & 1
\end{array}\right] \bullet\left[\begin{array}{cccc}
1 & 0 & 0 & 0 \\
0 & c \theta_{z_{0}} & -s \theta_{z_{0}} & 0 \\
0 & s \theta_{z_{0}} & c \theta_{z_{0}} & 0 \\
0 & 0 & 0 & 1
\end{array}\right] \\
& =\left[\begin{array}{cccc}
c \theta_{y_{0}} & s \theta_{y_{0}} s \theta_{z_{0}} & s \theta_{y_{0}} c \theta_{z_{0}} & 0 \\
s \theta_{x_{0}} s \theta_{y_{0}} & c \theta_{x_{0}} c \theta_{z_{0}}-s \theta_{x_{0}} c \theta_{y_{0}} s \theta_{z_{0}} & -c \theta_{x_{0}} s \theta_{z_{0}}-s \theta_{x_{0}} c \theta_{y_{0}} c \theta_{z_{0}} & 0 \\
-c \theta_{x_{0}} s \theta_{y_{0}} & s \theta_{x_{0}} c \theta_{z_{0}}+c \theta_{x_{0}} c \theta_{y_{0}} s \theta_{z_{0}} & -s \theta_{x_{0}} s \theta_{z_{0}}+c \theta_{x_{0}} c \theta_{y_{0}} c \theta_{z_{0}} & 0 \\
0 & 0 & 0 & 1
\end{array}\right]
\end{aligned}
$$


$\mathrm{x}-\mathrm{y}-\mathrm{z}$ is the initial position of the shoulder joint and the end position is:

$$
P=R \bullet T \bullet\left[\begin{array}{l}
x \\
y \\
z
\end{array}\right]
$$

\section{Sensor information collection and feedback}

The preliminary estimation of the total weight of the exoskeleton and upper limb and the length of upper arm is $10 \mathrm{~kg}$ and $250 \mathrm{~cm}$, respectively. The required torque of exoskeleton shoulder joint can then be estimated. The power of the shoulder joint drive motor was then selected to be $50 \mathrm{~W}$ and the drive motors for elbow and wrist joint were selected similarly. In a closed-loop control system, the selection of sensor and the feedback signal are very important. The encoder of shoulder and elbow joint can detect the position of the corresponding joint, and the 6-axis force sensor mounted on the wrist can detect the force and torque of wrist joint. Meanwhile, we mounted pressure sensor between the human upper arm, forearm and the exoskeleton upper arm, forearm to detect the force between the exoskeleton and human body.

In the early stage of rehabilitation treatment, the exoskeleton drives the motion of human upper limb by motors driving corresponding joints to the required position. When the treatment enters another stage, the upper limb has partial voluntary motions. By detecting the signals of the 6-axis force sensor we can determine if there is motion in shoulder or elbow. Combing with the pressure force signals of upper arm, forearm and position signals of each joint, we can get the movement of each joint.

\section{Discussion and conclusion}

The structure of the upper limb exoskeleton was designed by using differential mechanism of bevel gear transmission. As an exoskeleton with parallel actuated joints, it is highly rigid and accurate. The proposed exoskeleton also can reach a large workspace which is almost close toas the workspace that human upper limb can reach. Between every two rings, a trench structure was designed to place balls rather than using bearings, which greatly saved space, simplified the structure, and reduced the cost. Moreover a complex structure was used to unify the transmission ratio of each DOF. The simplification of the structure and the design a reasonable control system will be the next step of the research.

\section{Acknowledgement}

The work reported in this paper is funded by National High Technology Research and Development Program of China (863 Program) under Grant 2012AA041505 and the project of the State Key Laboratory of Robotics and System (SKLRS201201A02). 


\section{References}

[1] S. Cho, J. Ku et al., Development of virtual reality proprioceptive rehabilitation system for stroke patients, Computer Method and Programs in Biomedicine 113 (2014), 258-265.

[2] H.-M. Huang, J.-G. Liu and S.-Y. Gao, A virtual human modeling method for real-time movement and skin deformation, J. Computer Applications 24 (2004), 111-113.

[3] S.E. Fasoli, M. Fragala-Pinkham et al., Upper limb robot-assisted therapy: A new option for children with hemiplegia, Technology and Disability 22 (2010), 193-198.

[4] A. Gupta and M.K. O’Malley, Design of a haptic arm exoskeleton for training and rehabilitation, IEEE/ASME Trans. Mechatronics 11 (2006), 280-289.

[5] J. Klein, S.J. Spencer, J. Allington, K. Minakata, E.T. Wolbrecht, R. Smith, J.E. Bobrow and D.J. Reinkensmeyer, Biomimetic orthosis for the neurorehabilitation of the elbow and shoulder (BONES), Proc. of IEEE/ RAS-EMBS Int. Conf. on Biomedical Robotics and Biomechatronics 11 (2008), 535-541.

[6] D. Sasaki, T. Noritsugu and M. Takaiwa, Development of active support splint driven by pneumatic soft actuator (ASSIST), J. Robotics and Mechatronics 16 (2004), 497-502.

[7] J.F. Z., H.L. F., Y.M. D., Y. Z., C.J. Y. and Y. C., Novel 6DOF wearable exoskeleton arm with pneumatic forcefeedback for bilateral teleoperation, Chinese Journal of Mechanical Engineering 22 (2009).

[8] H.I. Krebs, B.T. Volpe, M.L. Aisen et al., Increasing productivity and quality of care: Robot-aided neuro-rehabilitation, Journal of Rehabilitation Research and Development 37 (2000), 639-652.

[9] K. Kiguchi, K. Iwami, M. Yasuda, K. Watanabe and T. Fukuda, An Exoskeletal robot for human shoulder joint motion assist, IEEE/ASME Trans. on Mechatronics 8 (2003), 125-135.

[10] K. Kiguchi, Active exoskeletons for upper limb motion assist, J. Humanoid Robotics 4 (2007), 607-624.

[11] J.C. Perry, J. Rosen and S. Burns, Upper limb powered exoskeleton design, IEEE/ASME Trans. on Mechatronics 12 (2007), 408-417.

[12] R.A.R.C. Gopura, K. Kiguchi and D.S.V. Bandara, A brief review on upper extremity robotic exoskeleton systems, International Conference on Industrial and Information Systems, 2011, 347-348.

[13] H. Kobayashi and K. Hiramatsu, Development of muscle suit for upper limb, Proc. IEEE Int. Conf. on Robotics and Automat. 3 (2004), 2480-2485.

[14] K. Meadmore, A.-M. Hughes et al., Functional electrical stimulation mediated by iterative learning control and 3D robotics reduces motor impairment in chronic stroke, Journal of NeuroEngineering and Rehabilitation 9 (2012), 2.

[15] J.F. Zhang, Exoskeleton based man machine intelligent system and its application, Ph.D. Dissertation, Zhejiang University, 2009.

[16] L.S. Lippert, Clinical kinesiology and anatomy, Davis Company, Philadelphia, 2006.

[17] F.H. Martini, M.J. Timmons and R.B. Tallitsch, Human anatomy, Prentice Hall, Pearson Education, $2003,8$.

[18] A.E. Engin, On the biomechanics of the shoulder complex, J. Biomech. 13 (1980), 575-590.

[19] B. Bolsterlee, D.H.E.J. Veeger and E.K. Chadwick, Clinical applications of musculoskeletal modeling for the shoulder and upper limb, Med. Biol. Eng. Comput. 51 (2013), 954.

[20] R.A.R.C. Gopura and K. Kiguchi, Mechanical designs of active upper limb exoskeleton robots state-of-the-art and design difficulties, 2009 IEEE 11th International Conference on Rehabilitation Robotics, 2009, 178-179.

[21] W. Maurel, D. Thalmann, P. Hoffmeyer et al., A biomechanical musculoskeletal model of human upper limb for dynamic simulation, Proceedings of the Eurographics Workshop on Computer Animation and Simulation, 1999, 121-136.

[22] J. de wen, J.C. Zhang et al., Rehabilitation engineering and biology mechanics, Tsinghua University Press, Beijing, pp. $307-320$.

[23] J.L. Cpons, Wearable Robots: Biomechatronic Exoskeletons, SoutherGate, Chichester, John Wiley \& Sons Ltd, England, 2008.

[24] C.P. Neu, J.J. Crisco and S.W. Wolfe, In vivo kinematic behavior of the radio-capitate joint during wrist flexionextension and radio-ulnar deviation, J. Biomech. 34 (2001), 1429-1438. 